\title{
A Hierarchical Algorithm for Integrated Scheduling and Control With Applications to Power Systems
}

Sokoler, Leo Emil; Dinesen, Peter Juhler; Jørgensen, John Bagterp

Published in:

IEEE Transactions on Control Systems Technology

Link to article, DOI:

10.1109/TCST.2016.2565382

Publication date:

2016

Document Version

Peer reviewed version

Link back to DTU Orbit

Citation (APA):

Sokoler, L. E., Dinesen, P. J., \& Jørgensen, J. B. (2016). A Hierarchical Algorithm for Integrated Scheduling and Control With Applications to Power Systems. IEEE Transactions on Control Systems Technology, 25(2), 590599. https://doi.org/10.1109/TCST.2016.2565382

\section{General rights}

Copyright and moral rights for the publications made accessible in the public portal are retained by the authors and/or other copyright owners and it is a condition of accessing publications that users recognise and abide by the legal requirements associated with these rights.

- Users may download and print one copy of any publication from the public portal for the purpose of private study or research.

- You may not further distribute the material or use it for any profit-making activity or commercial gain

- You may freely distribute the URL identifying the publication in the public portal 


\title{
A Hierarchical Algorithm for Integrated Scheduling and Control with Applications to Power Systems
}

\author{
Leo Emil Sokoler, Peter Juhler Dinesen, John Bagterp Jørgensen
}

\begin{abstract}
The contribution of this paper is a hierarchical algorithm for integrated scheduling and control via model predictive control (MPC) of hybrid systems. The controlled system is a linear system composed of continuous control, state, and output variables. Binary variables occur as scheduling decisions in the optimal control problem (OCP). The scheduling decisions are made on a slow time-scale compared to the system dynamics. This gives rise to a temporal separation of the scheduling and control variables in the OCP. Accordingly, the proposed hierarchical algorithm consists of two optimization levels. The upper level (scheduling level) solves a mixed-integer linear program (MILP) with a low frequency. The lower level (control level) solves a linear program (LP) with a high frequency. The main advantage of the proposed approach is that it requires online solution of an LP rather than an MILP. Simulations based on a power portfolio case study show that the hierarchical algorithm reduces the computation to solve the OCP by several orders of magnitude. The improvement in computation time is achieved without a significant increase in the overall cost of operation.
\end{abstract}

Index Terms-Model Predictive Control, Mixed-Integer Linear Programming, Production Scheduling, Hybrid Power Systems.

\section{INTRODUCTION}

Model predictive control (MPC) has become one of the most popular industrial control strategies [1]-[5]. The basic idea of MPC is to optimize the predicted behavior of a process model over a finite horizon. At each sampling instant, the current state is estimated based on measurements, and an optimal control problem (OCP) is formed and solved. The solution of the OCP provides a sequence of inputs. Only the first input in this sequence is applied to the controlled system, and the procedure is repeated at the following sampling instant. In this way, a closed-loop input trajectory is synthesized using feedback by solving a sequence of open-loop OCPs.

Control of hybrid systems is an emerging application area for MPC. Examples are traction control [6], control of refrigeration systems [7]-[9], control of co-generating power plants [10], water treatment control [11], and supply-chain management [12]. The main limitation of MPC for hybrid systems is that it requires solution of a computationally challenging OCP in real-time [13]-[17]. References [18]-[20] establish important properties for MPC of hybrid systems, such as closed-loop stability. Fault detection and state estimation in hybrid systems are described in [21].

Hybrid systems are often represented as mixed logical dynamical (MLD) systems [13], [22]-[26]. MLD systems are

The authors are affiliated with the Department of Applied Mathematics and Computer Science, Technical University of Denmark, DK-2800 Kgs. Lyngby, Denmark (corresponding author e-mail: jbjo@dtu.dk).

L. E. Sokoler is also affiliated with DONG Energy, DK-2830 Virum, Denmark. composed of continuous and binary inputs, states, outputs and auxiliary variables. The OCP that arises in MPC of MLD systems is a mixed-integer linear program (MILP) or a mixedinteger quadratic program (MIQP). Computationally tractable MPC schemes require algorithms that can solve the OCP in real-time. Efficient algorithms for MPC of MLD systems have been proposed in [13]-[17]. Reference [16] develops a structure-exploiting gradient projection algorithm for the subproblems that occur in a branch-and-bound algorithm for the OCP. References [13]-[15], [27] express the OCP as a multi-parametric MILP, which is solved off-line. The main issue with this explicit approach is that the computation time can grow exponentially with the problem size (horizon length, number of states, and number of inputs). Explicit methods are therefore usually limited to small-dimensional problems. Larger problems have been solved efficiently using convex relaxation techniques [28], [29], and Lagrangian decomposition [30], [31]. The performance of these methods is very problemdependent.

In this paper, we address a special case of MPC of MLD systems where decisions are made on two time scales. Binary scheduling decisions are made on a slow time-scale, while continuous control decisions are made on a fast time-scale. The novelty of this paper, is a hierarchical algorithm for solution of the OCP that occurs for this special case. The algorithm consists of an upper optimization level, which we refer to as the scheduling optimization level, and a lower optimization level, which we refer to as the control optimization level. The scheduling optimization level solves an MILP with a low frequency. The control optimization level solves a linear program (LP) with a high frequency. Binary decisions, made by the upper optimization level, are fixed in the lower optimization level. In the hierarchical algorithm, the time critical computation is solution of an LP. Without hierarchical decomposition of the MPC scheme, the time critical computation is solution of an MILP.

The main assumption of the proposed approach is that the binary decisions are made on a slow time-scale compared to the system dynamics. The application of the decomposition algorithm is therefore limited to solve OCPs that satisfy this assumption. However, such systems are ubiquitous in integrated scheduling and control [32]-[36]. Reference [37] shows that a range of production management problems related to control of chemical processes, e.g. batch operations, blending operations, and supply-chain optimization, fits well into the proposed framework. Motion planning problems in robotics are another application area that involves decisions on two time scales (geometric path planning and real-time feedback 
control) [38], [39]. The hierarchical separation of the scheduling layer and the control layer is similar to the separation of the optimization layer (RTO) and the control layer (MPC) commonly used in the chemical process industries [40]-[43]. The main difference is that the scheduling problem is a dynamic optimization problem involving both continuous and discrete variables, while the RTO is a steady-state optimization problem involving continuous variables only.

This work is motivated by the application of economic MPC (EMPC) to integrated scheduling and control in power system operations [35], [44]. In EMPC, the OCP objective function is directly related to the cost of operation [45]. For this reason, we focus on OCPs with a linear cost function rather than a conventional setpoint-based quadratic cost function. The proposed approach generalizes to OCPs with a quadratic cost function as well. As an illustrative example, we consider a power portfolio case study. The case study involves unit commitment and economic dispatch of a collection of power generators. An MPC scheme is employed for cost-efficient control of the power generators. The MPC scheme integrates production scheduling and balance control [46]. On/off decisions occur as binary variables in the OCP. Direct solution of the OCP is therefore intractable in real-time. Simulations show that the proposed hierarchical algorithm reduces the computation time to solve the OCP by several orders of magnitude. The improvement in computation time is achieved without a significant increase in the overall cost of operation. The algorithm also establishes a formal relationship between the OCP and the well known unit commitment (UC) problem [34], [47], [48], which has not previously been described in the literature. Related problems where a similar approach can be applied are e.g. control of co-generation power plants [10], [23], control of wind-farms for power optimization [49], and utility systems in the chemical process industries [34], [35], [50].

\section{A. Paper Organization}

This paper is organized as follows. Section II defines the OCP for integrated scheduling and control. Section III presents the hierarchical algorithm for efficient solution of the OCP. Section IV introduces the power portfolio problem. Section V provides simulations and results, and Section VI concludes the paper.

\section{Problem Definition}

We consider continuous-time linear state space models in the form

$$
\begin{aligned}
\dot{x}(t) & =A_{c} x(t)+B_{c} u(t)+E_{c} d(t), \\
z(t) & =C_{z} x(t)+F_{z} d(t) .
\end{aligned}
$$

The state-space matrices are denoted $\left(A_{c}, B_{c}, E_{c}, C_{z}, F_{z}\right)$, the control variable is denoted $u(t): \mathbb{R} \mapsto \mathbb{R}^{n_{u}}$, the system state is denoted $x(t): \mathbb{R} \mapsto \mathbb{R}^{n_{x}}$, the disturbance is denoted $d(t)$ : $\mathbb{R} \mapsto \mathbb{R}^{n_{d}}$, and the output is denoted $z(t): \mathbb{R} \mapsto \mathbb{R}^{n_{z}}$. MPC is applied to control the system (1). The prediction horizon is $T=\left[t_{0}, t_{f}\right]$.

\section{A. Optimal Control Problem}

This section defines the OCP for integrated scheduling and control. The OCP includes binary scheduling variables and continuous control variables. We partition the prediction horizon, $T$, into $L$ equidistant subintervals $T_{l}=\left[\tau_{l}, \tau_{l+1}\right]$, $l=0,1, \ldots, L-1$, such that $T=T_{0} \cup T_{1} \cup \cdots \cup T_{L-1}$. The length of each subinterval is $\Delta \tau=\left(t_{f}-t_{0}\right) / L . \tau_{0}=t_{0}$ and $\tau_{L}=t_{f}$. Let $\mathcal{L}=\{0,1, \ldots, L\}$ denote the set of indices associated with time instants $\tau_{0}, \tau_{1}, \ldots, \tau_{L}$. A vector of binary scheduling variables, $b_{l} \in\{0,1\}^{q}$, is associated with each time step, $\tau_{l}$.

The OCP is defined as

$$
\begin{array}{rlr}
\min _{X(\cdot), v(\cdot), b} & f_{\mathbb{R}}(X, v)+f_{\mathbb{Z}}(b), & \\
\text { s.t. } & \dot{x}(t)=A_{c} x(t)+B_{c} u(t)+E_{c} d(t), & t \in T, \\
& z(t)=C_{z} x(t)+F_{z} d(t), & t \in T, \\
& c_{\mathbb{R}}(X(t), v(t), t) \leq 0, & \\
& c_{\mathbb{Z}}(b) \leq 0, & \\
& \gamma(v(t), b)=0, & t \in T,
\end{array}
$$

The decision variables in (2) are the continuous-time functions $X(t)=\left[\begin{array}{lll}u(t)^{T} & x(t)^{T} & z(t)^{T}\end{array}\right]^{T}: \mathbb{R} \mapsto \mathbb{R}^{n_{u}+n_{x}+n_{z}}$ and $v(t): \mathbb{R} \mapsto \mathbb{R}^{q}$, and the vector of binary variables

$$
b=\left[\begin{array}{llll}
b_{0}^{T} & b_{1}^{T} & \cdots & b_{L}^{T}
\end{array}\right]^{T} .
$$

In general, $X(t)$ may also contain auxiliary continuous-time functions. Constraints (2b) and (2c) are the state-space constraints. The initial state, $x(0)=x_{0}$, is a fixed parameter. Equation (2d) represents continuous-time constraints. These constraints are related to the continuous control decisions for the system (1). Equation (2e) represents discrete-time constraints. These constraints are related to the binary scheduling decisions for the system (1). We assume that $c_{\mathbb{R}}$ and $c_{\mathbb{Z}}$ are affine functions.

In the objective function $(2 \mathrm{a}), f_{\mathbb{R}}(X, v)$ is the cost associated with the continuous control decisions, and $f_{\mathbb{Z}}(b)$ is the cost associated with the binary scheduling decisions. We assume that $f_{\mathbb{R}}$ and $f_{\mathbb{Z}}$ are linear functions. Moreover

$$
f_{\mathbb{R}}(X, v)=\int_{t_{0}}^{t_{f}} g_{\mathbb{R}}(X(t), v(t), t) d t .
$$

Constraint (2f) couples the vector of binary variables, $b$, and the continuous-time function $v(t)$. The coupling function, $\gamma$, is defined as

$$
\gamma(v(t), b)= \begin{cases}v(t)-b_{0} & \text { if } \tau_{0} \leq t \leq \tau_{1} \\ v(t)-b_{L-1} & \text { if } \tau_{L-1} \leq t \leq \tau_{L} .\end{cases}
$$

This means that any feasible solution of (2) satisfies

$$
v(t)=b_{l}, \quad \tau_{l} \leq t \leq \tau_{l+1},
$$

for $l \in \mathcal{L} \backslash\{L\}$. The main purpose of the coupling function, $\gamma$, is to decouple the binary and continuous variables in $c_{\mathbb{R}}$ and $c_{\mathbb{Z}}$, as well as in $f_{\mathbb{R}}$ and $f_{\mathbb{Z}}$. Note that the system dynamics, (2b), does not depend on the binary variables. 


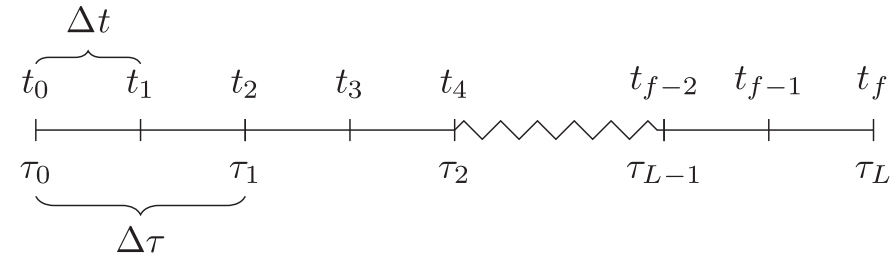

Fig. 1. The two time scales associated with the OCP (7). The sampling time of the continuous-time system, (1), is $\Delta t$. The time between the binary decisions, (3), is $\Delta \tau$. This paper addresses the case where $\Delta t$ is small compared to $\Delta \tau$.

\section{B. Discretization}

To solve (2), we discretize the optimization problem. The sampling time is $\Delta t=\Delta \tau / K$, for some positive integer $K$. The discretization points are denoted $t_{0}, t_{1}, \ldots, t_{N}$. We let $\mathcal{N}=\{0,1, \ldots, N\}$ denote the set of indices associated with the discretization points. Note that

$$
\tau_{l}=t_{K l}, \quad l \in \mathcal{L} .
$$

Fig. 1 illustrates the relation (6) for $K=2$.

We consider the case where binary decisions are made on a slow time-scale, compared to the system dynamics. This means that $\Delta t$ is small compared to $\Delta \tau$, i.e. $N \gg L$. The discrete-time formulation of (2) is

$$
\begin{aligned}
& \min _{X, v, b} \tilde{f}_{\mathbb{R}}(X, v)+f_{\mathbb{Z}}(b), \\
& \text { s.t. } x_{k+1}=A x_{k}+B u_{k}+E d_{k}, \quad k \in \mathcal{N} \backslash\{N\} \text {, } \\
& z_{k}=C_{z} x_{k}+F_{z} d_{k}, \quad k \in \mathcal{N} \backslash\{0\}, \\
& c_{\mathbb{R}}\left(X_{k}, v_{k}, t_{k}\right) \leq 0, \quad k \in \mathcal{N}, \\
& c_{\mathbb{Z}}(b) \leq 0 \text {, } \\
& \gamma\left(v_{k}, b\right)=0 \text {, } \\
& k \in \mathcal{N} .
\end{aligned}
$$

Constraints (7b) and (7c) are the discrete-time equivalents of (2b) and (2c), respectively. The discrete-time state-space matrices are denoted $A, B$, and $E . u_{k} \in \mathbb{R}^{n_{u}}$ is the control variable, $x_{k} \in \mathbb{R}^{n_{x}}$ is the system state, $d_{k} \in \mathbb{R}^{n_{d}}$ is the disturbance, and $z_{k} \in \mathbb{R}^{n_{z}}$ is the output. The initial state, $x_{0}$, is a fixed parameter. As in the continuous-time case, we use $X_{k}=\left[\begin{array}{lll}u_{k}^{T} & x_{k}^{T} & z_{k}^{T}\end{array}\right]^{T}$ for compact notation. This vector may also contain additional auxiliary variables. In the objective function (7a), we have defined

$$
\tilde{f}_{\mathbb{R}}(X, v)=\sum_{k \in \mathcal{N} \backslash\{N\}} g_{\mathbb{R}}\left(X_{k}, v_{k}, t_{k}\right) \Delta t,
$$

which is an Euler approximation of the integral (4).

\section{HiERARCHICAL Algorithm}

Problem (7) is an MILP. Solving (7) in real-time is therefore challenging, especially since $N$ is large. To reduce the computation time to solve (7), we consider a hierarchical approach. The idea is to decompose the solution of (7) into two separate optimization levels. The upper optimization level (scheduling level) is associated with the binary decisions that are made on a slow time-scale, and the lower optimization level (control level) is associated with the control decisions that are made on a fast time-scale. Binary variables are fixed at the lower optimization level. For this reason, the lower level optimization problem can be expressed as an LP. We refer to the upper level MILP as the UL-OCP, and to the lower level LP as the LLOCP. The UL-OCP is solved with a low frequency, and the the LL-OCP is solved with a high frequency.

\section{A. Upper Level Optimal Control Problem (UL-OCP)}

The UL-OCP is simply (7), where we replace the sampling time, $\Delta t$, with some $\Delta \bar{t}$ that satisfies

$$
\Delta t \leq \Delta \bar{t} \leq \Delta \tau
$$

with $\Delta \tau / \Delta \bar{t}$ integer. We use bar notation to denote the variables and the parameters associated with the UL-OCP. In case $\Delta \bar{t}=\Delta t$, the UL-OCP coincides with (7). When $\Delta \bar{t}=\Delta \tau$, the sampling time is the time between binary decisions. By assumption, the system dynamics occurs at a much faster time scale than $\Delta \tau$. Therefore, the state transition matrix, $\bar{A}$, resulting from a discretization with $\Delta \bar{t}=\Delta \tau$, satisfies $\bar{A} \approx 0$. In addition

$$
\bar{z}_{k} \approx C_{z}\left(\bar{B} \bar{u}_{k-1}+\bar{E} \bar{d}_{k-1}\right)+F_{z} \bar{d}_{k}, \quad k \in \mathcal{N} .
$$

Consequently, the state variables $\bar{x}_{1}, \bar{x}_{2}, \ldots, \bar{x}_{\bar{N}}$ can be eliminated from the UL-OCP, for a sufficiently large $\Delta \bar{t}$.

\section{B. Lower Level Optimal Control Problem (LL-OCP)}

The LL-OCP is (7) with fixed binary variables, $b=\tilde{b}$. As the binary variables are fixed, we do not include $f_{\mathbb{Z}}(\tilde{b})$ in the LLOCP objective function. Similarly, constraint (7e) is excluded from the LL-OCP. The LL-OCP is

$$
\begin{aligned}
& \min _{X} \sum_{k \in \tilde{\mathcal{N}} \backslash\{\tilde{N}\}} g_{\mathbb{R}}\left(X_{k}, \tilde{v}_{k}, t_{k}\right) \Delta t, \\
& \text { s.t. } x_{k+1}=A x_{k}+B u_{k}+E d_{k}, \quad k \in \tilde{\mathcal{N}} \backslash\{\tilde{N}\} \text {, } \\
& z_{k}=C_{z} x_{k}+F_{z} d_{k}, \quad k \in \tilde{\mathcal{N}} \backslash\{0\}, \\
& c_{\mathbb{R}}\left(X_{k}, \tilde{v}_{k}, t_{k}\right) \leq 0, \quad k \in \tilde{\mathcal{N}},
\end{aligned}
$$

where $\tilde{\mathcal{N}}=\{0,1, \ldots, \tilde{N}\}$. The variables $v_{1}=\tilde{v}_{1}, v_{2}=$ $\tilde{v}_{2}, \ldots, v_{N}=\tilde{v}_{N}$ are fixed parameters in (9). These parameters are determined by the coupling function (5), for fixed $b=\tilde{b}$. Since the system dynamics occurs on a relatively fast timescale, $\tilde{N}$ may be chosen significantly smaller than $N$.

\section{Algorithm}

Consider the stochastic system

$$
\begin{aligned}
\boldsymbol{x}_{k+1} & =A \boldsymbol{x}_{k}+B u_{k}+E \boldsymbol{d}_{k}+G \boldsymbol{w}_{k}, \\
\boldsymbol{z}_{k} & =C_{z} \boldsymbol{x}_{k}+F_{z} \boldsymbol{d}_{k}, \\
\boldsymbol{y}_{k} & =C_{y} \boldsymbol{x}_{k}+F_{y} \boldsymbol{d}_{k}+\boldsymbol{v}_{k},
\end{aligned}
$$

where $\boldsymbol{w}_{k} \sim N\left(0, R_{w}\right)$ is the process noise, $\boldsymbol{v}_{k} \sim N\left(0, R_{v}\right)$ is the measurement noise, and $\boldsymbol{d}_{k} \sim N\left(\breve{d}_{k}, R_{d}\right)$ is an unknown disturbance. We use bold letters to denote random variables.

Define $\mathcal{I}_{k}=\left\{\mathcal{I}_{k-1}, u_{k-1}, d_{k-1}, y_{k}\right\}$, with $\mathcal{I}_{0}=y_{0}$. Moreover, introduce the conditional means $\hat{x}_{k+j \mid k}=E\left[\boldsymbol{x}_{k+j} \mid \mathcal{I}_{k}\right]$, $\hat{y}_{k+j \mid k}=E\left[\boldsymbol{y}_{k+j} \mid \mathcal{I}_{k}\right], \hat{z}_{k+j \mid k}=E\left[\boldsymbol{z}_{k+j} \mid \mathcal{I}_{k}\right]$, and the conditional covariance matrix $P_{k+j \mid k}=V\left[\boldsymbol{x}_{k+j} \mid \mathcal{I}_{k}\right]$. These values 


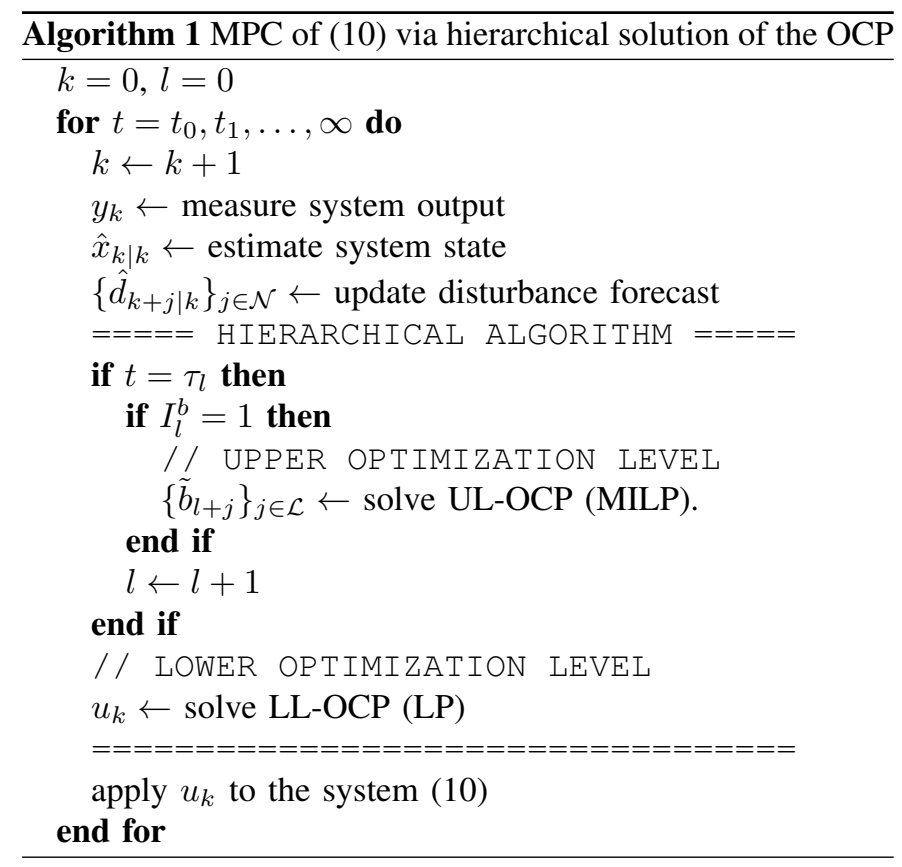

are computed using the Kalman filter. The disturbance forecast is denoted $\hat{d}_{k+j \mid k}$. We assume that a disturbance forecast is generated by some external procedure.

In MPC, based on the separation and certainty equivalence principle, uncertain parameters are replaced by their conditional expectations. We use this approach to control the stochastic system (10). The OCP solved at every sampling instant, $k=0,1, \ldots, \infty$, is (7), with $x_{0}=\hat{x}_{k \mid k}$, and $d_{j}=\hat{d}_{k+j \mid k}$ for $j \in \mathcal{N}$. Algorithm 1 is an MPC scheme for control of the stochastic system (10), using the hierarchical algorithm for solution of the OCP. The indicator variables $I_{1}^{b}, I_{2}^{b}, \ldots, I_{\infty}^{b}$ trigger solution of the UL-OCP. The indicator variables can be pre-defined or updated online, e.g. it is reasonable to update the scheduling decisions if a significant change in the disturbance forecast arises. The lower optimization level requires that values for the binary variables are available within its control horizon. Therefore, $I_{0}^{b}=1$.

There are several parameters in Algorithm 1 that can be tuned to trade-off optimality and computation time. Increasing the sampling time, $\Delta \bar{t}$, reduces the size of the UL-OCP. Reducing the length of the prediction horizon, $\tilde{N}$, reduces the size of the LL-OCP. As an example, $\tilde{N}$ may be chosen just large enough to ensure that the controller is stable [51]-[53]. We suggest to use a fairly long prediction horizon to achieve stable and cost-efficient operation of the system.

\section{Power Portfolio Problem}

Electricity is bought and sold in electricity markets. A majority of the energy is usually traded in a day-ahead energy market. When the market is cleared, the power producers receive a 24 hours-ahead reference profile specifying the amount of electricity they have sold. Scheduling the generation of available power generators is a challenging task for the power producers, as it involves decisions across different time scales and scheduling horizons. Hours-ahead to days-ahead scheduling (scheduling level) is handled by solving an MILP for unit commitment and economic dispatch of the power generators. To account for imbalances between the power production and the reference profile, a balance control layer (control level) can be employed. The control level is important to account for the inherent uncertainties associated with the generation of renewable energy sources [44], [46]. We show that the proposed approach facilities the integration of the scheduling and the control level. This makes it possible to trade-off computation time and optimality in a systematic way.

A collection of $M$ power generators is controlled using the proposed MPC scheme. The $M$ power generators represent a portfolio of generators that is operated by a single power producer. The generators are modeled as transfer functions in the form

$$
Z_{i}(s)=G_{u, i}(s) U_{i}(s), \quad i \in \mathcal{M},
$$

where $\mathcal{M}=\{1,2, \ldots, M\} . U_{i}(s)$ is the power production setpoint, and $Z_{i}(s)$ is the power production. We define $D(s)$ to be the aggregated power production from non-controllable generators, such as photovoltaic generators and non-controllable wind turbines. The net power production is the sum

$$
Z_{T}(s)=\sum_{i \in \mathcal{M}} Z_{i}(s)+D(s) .
$$

We collect (11) and (12) into a single model in the form

$$
Z(s)=G_{u}(s) U(s)+G_{d}(s) D(s),
$$

where $U(s)=\left[U_{1}(s)^{T}, \ldots, U_{M}(s)^{T}\right]^{T}$ is the control variable, and $Z(s)=\left[Z_{1}(s)^{T}, \ldots, Z_{M}(s)^{T}, Z_{T}(s)\right]^{T}$ is the output.

$$
G_{u}(s)=\left[\operatorname{blkdiag}\left(G_{u, 1}, \ldots, G_{u, M}\right) ;\left[G_{u, 1}, \ldots, G_{u, M}\right]\right]
$$

and $G_{d}(s)=[0, \ldots, 0,1]^{T}$ are the transfer functions. The model (13) is realized in the continuous-time state-space form (1). In the resulting model structure

$$
\begin{aligned}
& A_{c}=\operatorname{blkdiag}\left(A_{c, 1}, A_{c, 2}, \ldots, A_{c, M}\right), \\
& B_{c}=\operatorname{blkdiag}\left(B_{c, 1}, B_{c, 2}, \ldots, B_{c, M}\right), \\
& C_{z}=\left[\operatorname{blkdiag}\left(C_{z, 1}, \ldots, C_{z, M}\right) ;\left[C_{z, 1}, \ldots, C_{z, M}\right]\right],
\end{aligned}
$$

$E_{c}=0$, and $F_{z}=[0, \ldots, 0,1]^{T}$. Moreover, $x(t)$ is the system state, $u(t)=\left[u_{1}(t)^{T}, \ldots, u_{M}(t)^{T}\right]^{T}$ is the vector of power production setpoints, and $z(t)=\left[z_{1}(t)^{T}, \ldots, z_{M+1}(t)^{T}\right]^{T}$ is the vector of power production outputs. This vector contains the power production of each generator, as well as the total power production, $z_{M+1}(t)=z_{T}(t)$. Finally, $d(t)$ is the power production from non-controllable generators.

The power generators can be turned on and off in the time instants $t \in\left\{\tau_{0}, \tau_{1}, \ldots, \tau_{L}\right\}$. Define the binary variables

$$
b_{i, l}^{s}= \begin{cases}1 & \text { if generator } i \text { is running at time } \tau_{l} \\ 0 & \text { otherwise, }\end{cases}
$$

$i \in \mathcal{M}$ and $l \in \mathcal{L}$. Similarly, define $b_{i, l}^{u}$ to indicate if a generator is turned on, and $b_{i, l}^{d}$ to indicate if a generator is turned off. In addition

$$
v_{i}^{s}(t)=b_{i, l}^{s}, \quad \tau_{l} \leq t \leq \tau_{l+1},
$$


for $i \in \mathcal{M}$ and $l \in \mathcal{L} \backslash\{L\}$. I.e. $v_{i}^{s}(t)$ is a piecewise constant representation of $b_{i, l}^{s}$. We define $v_{i}^{u}(t)$ and $v_{i}^{d}(t)$ in the same manner.

The cost of operation is defined as

$$
\begin{gathered}
\psi=\int_{t_{0}}^{t_{f}}\left(\sum_{i \in \mathcal{M}}\left(p_{i}^{z} z_{i}(t)+p_{i}^{s} v_{i}^{s}(t)\right)+p^{\rho} \rho(t)\right) d t \\
+\sum_{i \in \mathcal{M}} \sum_{l \in \mathcal{L}}\left(p_{i}^{u} b_{i, l}^{u}+p_{i}^{d} b_{i, l}^{d}\right) .
\end{gathered}
$$

$p_{i}^{z}$ is the marginal production price for power generator $i$, $p_{i}^{s}$ is the fixed running cost for power generator $i, p_{i}^{u}$ is the start-up cost for power generator $i$, and $p_{i}^{d}$ is the shutdown cost for power generator $i . p^{\rho}$ is the marginal price for power imbalances. Imbalance costs are imposed when the power production deviates from a pre-defined reference (e.g. the power demand or the power sold in the day-ahead electricity market), denoted $r(t)$. This is expressed as

$$
r(t)-\rho(t) \leq z_{M+1}(t) \leq r(t)+\rho(t), \quad t \in T .
$$

To keep the notation simple, we use one single price, $p^{\rho}$, for both positive and negative imbalances. Asymmetric prices can represent a market situation where power is traded on an electricity exchange.

The power production setpoint for a generator is limited by its capacity limits. This is modeled by the constraint

$$
\underline{z}_{i} v_{i}^{s}(t) \leq u_{i}(t) \leq \bar{z}_{i} v_{i}^{s}(t), \quad i \in \mathcal{M}, t \in T .
$$

The lower and upper capacity limit for power generator $i$ is $\underline{z}_{i}$ and $\bar{z}_{i}$, respectively. The setpoint for a generator not running is zero. This is enforced by multiplying each of the capacity limits in (17) by $v_{i}^{s}(t)$. A constraint on the input-rate is defined to avoid drastic setpoint changes

$$
\partial \underline{u}_{i}-Q v_{i}^{d}(t) \leq \frac{d u_{i}(t)}{d t} \leq \partial \bar{u}_{i}+Q v_{i}^{u}(t),
$$

$i \in \mathcal{M}, t \in T$. The upper and lower input-rate limit for generator $i$ is $\partial \underline{u}_{i}$ and $\partial \bar{u}_{i}$, respectively. The terms involving $Q$ relax the constraint (18) when a generator is turned on or off. This is necessary to avoid infeasibility of the OCP, e.g. when $\underline{u}_{i} \geq \Delta \bar{u}_{i}$ for some $i \in \mathcal{M}$. The binary decision variables are coupled by the constraints

$$
\begin{array}{ll}
b_{i, l}^{s}=b_{i, l-1}^{s}+\left(b_{i, l}^{u}-b_{i, l}^{d}\right), & i \in \mathcal{M}, \quad l \in \mathcal{L}, \\
b_{i, l}^{u}+b_{i, l}^{d} \leq 1, & i \in \mathcal{M}, \quad l \in \mathcal{L} .
\end{array}
$$

These constraints model the start-up and shut-down logic. We refer to [47] for a detailed description of the logical constraints in unit commitment and economic dispatch problems. The OCP associated with the power portfolio problem is to minimize (15) subject to the constraints (16)-(19).

\section{A. Standard Form}

The OCP associated with the power portfolio problem is written in the standard form (7). The components of the binary vector, (3), are

$$
b_{l}=\left[\begin{array}{lllllll}
b_{1, l}^{s} & b_{1, l}^{u} & b_{1, l}^{d} & \ldots & b_{M, l}^{s} & b_{M, l}^{u} & b_{M, l}^{d}
\end{array}\right],
$$

for $l \in \mathcal{L}$. We define the components of $v$ accordingly. In the objective function (2a)

$$
\begin{aligned}
f_{\mathbb{R}}(X, v) & =\int_{t_{0}}^{t_{f}}\left(\sum_{i \in \mathcal{M}}\left(p_{i}^{z} z_{i}(t)+p_{i}^{s} v_{i}^{s}(t)\right)+p^{\rho} \rho(t)\right) d t, \\
f_{\mathbb{Z}}(b) & =\sum_{i \in \mathcal{M}} \sum_{l \in \mathcal{L}} p_{i}^{u} b_{i, l}^{u}+p_{i}^{d} b_{i, l}^{d},
\end{aligned}
$$

such that

$$
g_{\mathbb{R}}(X(t), v(t), t)=\sum_{i \in \mathcal{M}}\left(p_{i}^{z} z_{i}(t)+p_{i}^{s} v_{i}^{s}(t)\right)+p^{\rho} \rho(t),
$$

and $X(t)=\left[\begin{array}{llll}u(t)^{T} & x(t)^{T} & z(t)^{T} & \rho(t)^{T}\end{array}\right]^{T}$. The function associated with the constraint $(2 \mathrm{~d})$ is

$$
c_{\mathbb{R}}(X(t), v(t), t)=\left[\begin{array}{c}
\bar{c}_{\mathbb{R}}(X(t), v(t), t) \\
c_{\mathbb{R}}^{0}(X(t), v(t), t) \\
\vdots \\
c_{\mathbb{R}}^{N}(X(t), v(t), t)
\end{array}\right],
$$

where

$$
\begin{aligned}
& \bar{c}_{\mathbb{R}}(X(t), v(t), t)= {\left[\begin{array}{l}
z_{M+1}(t)-r(t)-\rho(t) \\
r(t)-z_{M+1}(t)-\rho(t)
\end{array}\right], } \\
& c_{\mathbb{R}}^{i}(X(t), v(t), t)=\left[\begin{array}{c}
u_{i}(t)-\bar{z}_{i} v_{i}^{s}(t) \\
\underline{z}_{i} v_{i}^{s}(t)-u_{i}(t) \\
\frac{d u_{i}(t)}{d t}-\Delta \bar{u}_{i}-Q v_{i}^{u}(t) \\
\Delta \underline{u}_{i}-Q v_{i}^{d}(t)-\frac{d u_{i}(t)}{d t}
\end{array}\right], \quad i \in \mathcal{M} .
\end{aligned}
$$

$c_{\mathbb{Z}}(b)$ is constructed by stacking the constraints (19). The coupling constraint (2f) follows from the definition (5).

In the discretized OCP, (7), we use the backward-difference approximation

$$
\frac{d u_{i}(t)}{d t} \approx \frac{u_{i, k}-u_{i, k-1}}{\Delta t}, \quad i \in \mathcal{M}, \quad k \in \mathcal{N} .
$$

The constraints for the discretized OCP are expressed as in (20) with

$$
\begin{aligned}
& \bar{c}_{\mathbb{R}}\left(X_{k}, v_{k}, t_{k}\right)= {\left[\begin{array}{l}
z_{M+1, k}-r_{k}-\rho_{k} \\
r_{k}-z_{M+1, k}-\rho_{k}
\end{array}\right], } \\
& c_{\mathbb{R}}^{i}\left(X_{k}, v_{k}, t_{k}\right)=\left[\begin{array}{c}
u_{i, k}-\bar{z}_{i, k} v_{i, k}^{s} \\
\underline{z}_{i} v_{i, k}^{s}-u_{i, k} \\
\frac{u_{i, k}-u_{i, k-1}}{\Delta t}-\partial \bar{u}_{i}-Q v_{i, k}^{u} \\
\partial \underline{u}_{i}-Q v_{i, k}^{d}-\frac{u_{i, k}-u_{i, k-1}}{\Delta t}
\end{array}\right], \quad i \in \mathcal{M} .
\end{aligned}
$$

The discretized OCP is an MILP. To get well-behaved closedloop solutions, the OCP objective function can be augmented by $\ell_{1}$ - and $\ell_{2}$-penalty terms on the input-rate [54]. For $\ell_{2}$ regularization, the OCP becomes an MIQP. It is straightforward to generalize the hierarchical algorithm to this case.

\section{B. Relationship to the Unit Commitment Problem}

The transfer function (11) maps the power production setpoint for a generator to its actual power production. It is reasonable to assume that the gain in this system is 1 . This means that

$$
\begin{aligned}
z_{i}(t) & \rightarrow u_{i}(t), \\
z_{M+1}(t) & \rightarrow \sum_{i \in \mathcal{M}} u_{i}(t)+d(t),
\end{aligned}
$$


TABLE I

CASE STUDY GENERATOR PARAMETERS.

\begin{tabular}{llll}
\hline Parameter/Generator & Gen. 1 & Gen. 2 & Gen. 3 \\
\hline$\kappa_{i}[\mathrm{~s}]$ & 20 & 25 & 40 \\
$p_{i}^{z}[\mathrm{EUR} / \mathrm{MWh}]$ & 80 & 40 & 10 \\
$p_{i}^{s}[\mathrm{EUR} / \mathrm{h}]$ & 25 & 10 & 5 \\
$p_{i}^{u}[\mathrm{EUR}]$ & 100 & 150 & 200 \\
$p_{i}^{d}[\mathrm{EUR}]$ & 0 & 0 & 0 \\
$\left(\partial \underline{u}_{i}, \partial \bar{u}_{i}\right)[\mathrm{MW} / \mathrm{s}]$ & $(-0.2,0.2)$ & $(-0.1,0.1)$ & $(-0.05,0.05)$ \\
$\left(\underline{z}_{i}, \bar{z}_{i}\right)[\mathrm{MW}]$ & $(0.5,5)$ & $(2,10)$ & $(5,25)$ \\
\hline
\end{tabular}

for $t \rightarrow \infty$. Consider the approximation (8) for the UL-OCP. Based on (21), we can further assume that

$$
C_{z} \bar{B} \approx[\operatorname{blkdiag}(I, \ldots, I) ;[1,1,1]],
$$

where we use that $E=0$ and $F_{z}=[0, \ldots, 0,1]^{T}$. Consequently

$$
\left[\begin{array}{c}
\bar{z}_{1, k} \\
\vdots \\
\bar{z}_{M, k} \\
\bar{z}_{T, k}
\end{array}\right]=\left[\begin{array}{c}
\bar{u}_{1, k-1} \\
\vdots \\
\bar{u}_{M, k} \\
\sum_{i \in \mathcal{M}} \bar{u}_{i, k}
\end{array}\right]+\left[\begin{array}{c}
0 \\
\vdots \\
0 \\
\bar{d}_{k}
\end{array}\right], \quad k \in \mathcal{N} .
$$

Equation (22) is used to eliminate the power production variables, $\bar{z}_{1}, \bar{z}_{2}, \ldots, \bar{z}_{\bar{N}}$ from the UL-OCP. In this way, the UL-OCP can be expressed without state and output variables, as well as without the state-space constraint (7b) and (7c). The resulting optimization problem is a UC problem [34], [47], [48]. I.e. for a sufficiently coarse temporal discretizaton, the UL-OCP coincides with the UC problem. Hierarchical decomposition of unit commitment and balance control is widely adopted in power system operations [34], [48]. This paper shows that the hierarchical approach can be interpreted as an approximate way to solve the OCP (7). The approximation provides a computationally efficient scheme to obtain suboptimal solutions of (7). This makes it possible to employ MPC, based on (7), for integrated scheduling and control.

\section{CAse Study}

We consider an example of the power portfolio problem, with $M=3$ generators in the form

$$
G_{u, i}(s)=\frac{1}{\left(1+\kappa_{i} s\right)^{3}}, \quad i \in \mathcal{M} .
$$

Reference [55] validates the model (23) against actual measurement data. Note that the gain in the system (23) is 1. The controlled system is a stochastic system in the form (10). The disturbance, $\boldsymbol{d}_{k}$, is the non-controllable wind power production. For this case study, we do not consider process noise nor measurement noise.

Table I lists case study parameters for each of the three generators in convenient display units. Generator 1 has a small time constant, i.e. the generator is fast. It has a high marginal production price and a high fixed running cost. Moreover, Generator 1 has a very limited capacity. In contrast to this, Generator 3 is a slow low-cost generator with a large capacity. Generator 2 is a medium-sized generator. The contrast between generator agility and production price is a common situation in

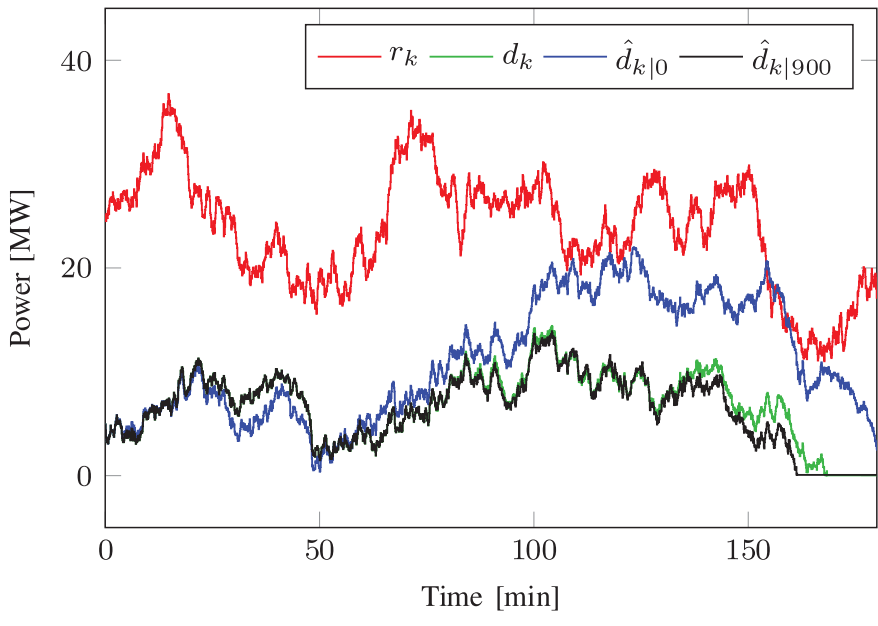

Fig. 2. Case study power demand (reference, $r_{k}$ ), wind power production (disturbance, $d_{k}$ ), and wind power forecasts (disturbance forecasts, $\hat{d}_{k \mid 0}$ and $\left.\hat{d}_{k \mid 900}\right)$.

power systems where large thermal power plants often produce a majority of the electricity, while the use of smaller gas turbines is limited to critical peak periods.

The imbalance price, $p^{\rho}$, is $400 \mathrm{EUR} / \mathrm{MWh}$. The time between binary decisions is $\Delta \tau=900 \mathrm{~s}$. This means that the generators can be turned on or off every 15 minutes. The length of the prediction horizon is $t_{f}=3$ hours. The sampling time for the system dynamics is $\Delta t=5 \mathrm{~s}$, which is adequate for dynamics in the time scale listed in Table I. As a result of these parameter specifications, $N=2160$, and $L=12$. Unit commitment is often performed with a prediction horizon of more than 24 hours. We use a 3 hours-ahead horizon to be able to solve the full size OCP (7) using a general-purpose solver in a reasonable amount of time. Fig. 2 illustrates the case study reference, $r_{k}$, the wind power production, $d_{k}$, the initial wind power production forecast, $\hat{d}_{k \mid 0}$, and an updated wind power production forecast, $\hat{d}_{k \mid 900}$. The wind power forecasts should be interpreted as $\left\{\hat{d}_{k \mid 0}\right\}_{k=0}^{2160}$ and $\left\{\hat{d}_{k \mid 900}\right\}_{k=0}^{2160}$, respectively. Note that $\hat{d}_{k \mid 900}=d_{k}$ for $k \leq 900$. For $t>75 \min (k>900)$, the initial wind power forecast, $\hat{d}_{k \mid 0}$, is not very accurate. This suggests that the UL-OCP should be resolved when the updated forecast, $\hat{d}_{k \mid 900}$, becomes available at $t=75 \mathrm{~min}$ $(k=900)$.

\section{A. Known Disturbance}

Assume that the disturbance, $\boldsymbol{d}_{k}$, is known over the entire 24-hour prediction horizon. Fig. 3 illustrates the open-loop production plan obtained by direct solution of the OCP (7). Fig. 3 (d) shows that the total power production follows the reference well. Large imbalance costs are therefore avoided when $\boldsymbol{d}_{k}$ is known. The total cost of operation is EUR 2426.

The computation time to solve the OCP (7) is approximately 15 min using Gurobi. Direct solution of (7) is thus intractable in real-time. To overcome this issue, (7) is solved using Algorithm 1. We consider a situation with a fixed horizon length, $\tilde{N}=2160$, in the LL-OCP, and varying sampling time, $\Delta \bar{t}$, in the UL-OCP. Average values are used for $\bar{r}_{k}$ and $\bar{d}_{k}$ 


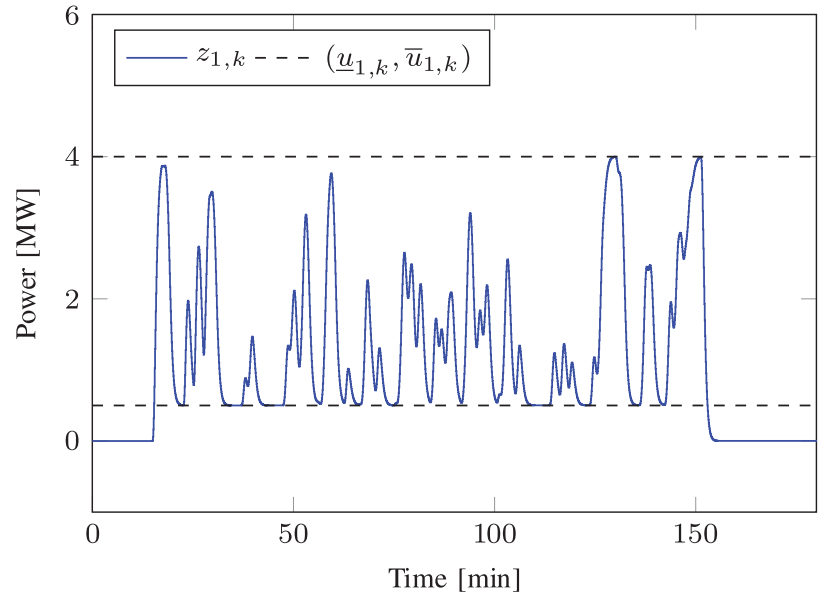

(a) Power production level: Generator 1.

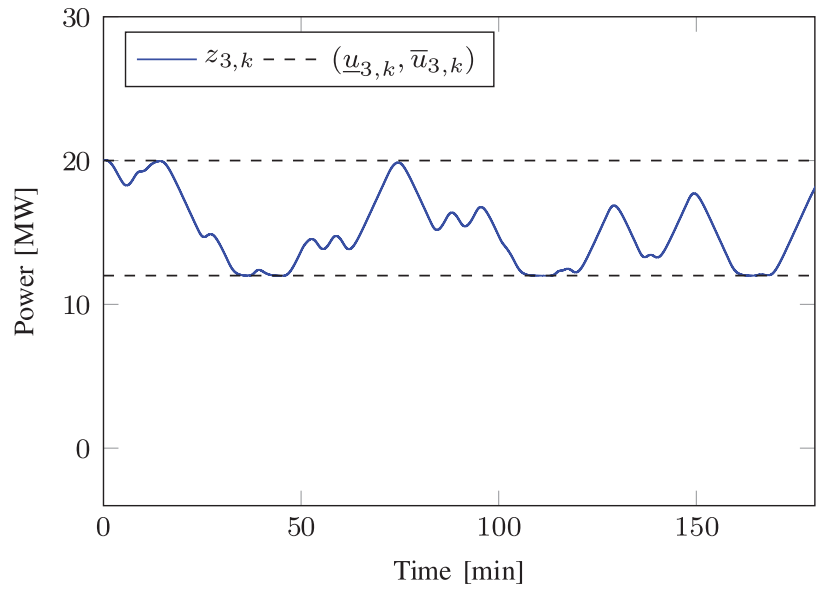

(c) Power production level: Generator 3.

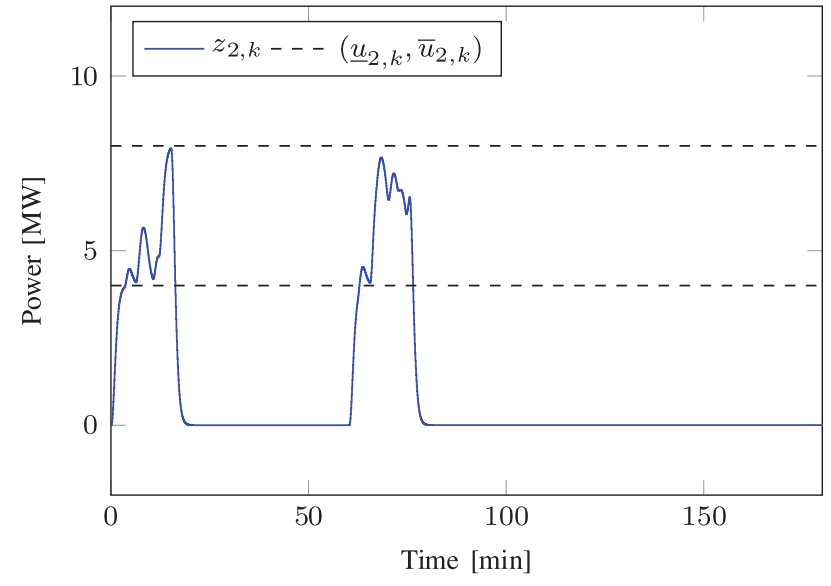

(b) Power production level: Generator 2 .

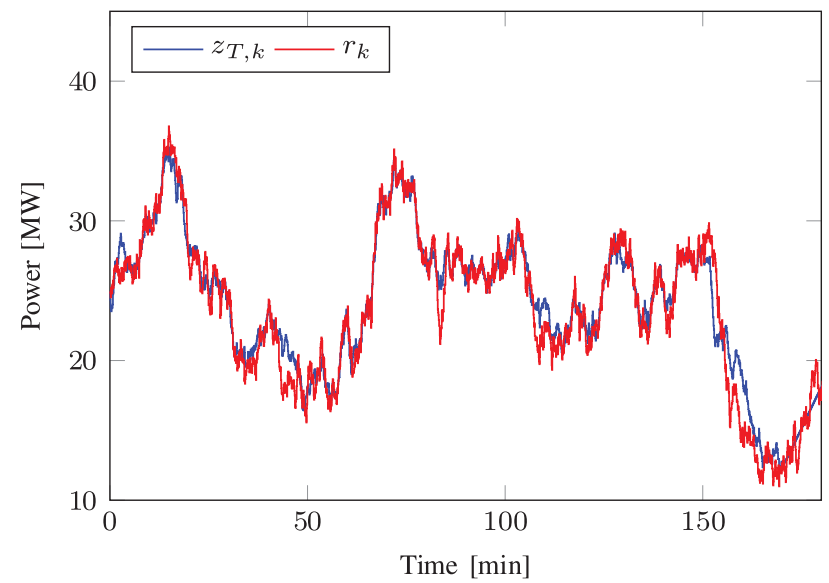

(d) Total power production.

Fig. 3. Open-loop simulation: Generator power production levels and total power production.

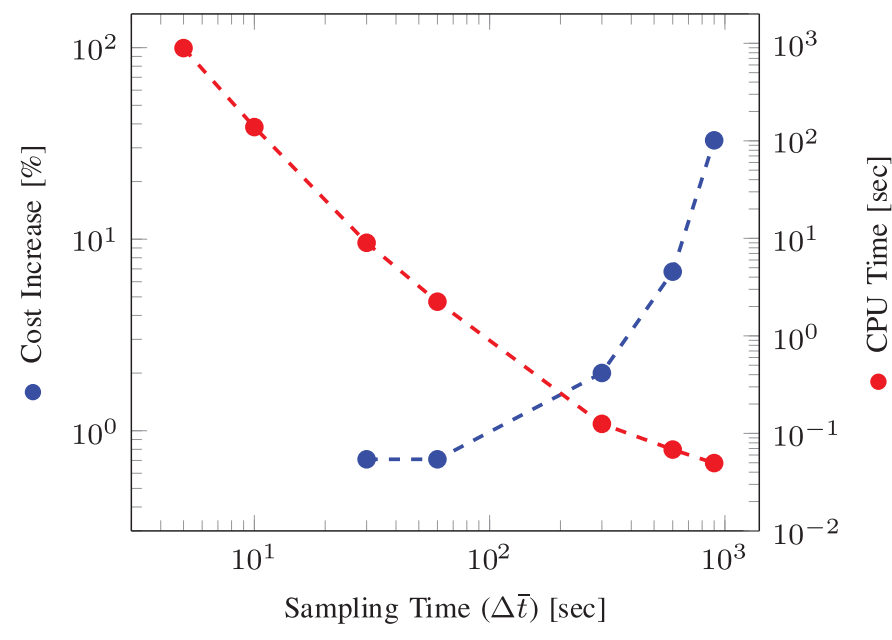

Fig. 4. Relative cost increase and computation time to solve the UL-OCP with $t_{f}=3$ hours, as a function of the sampling time $\Delta \bar{t}$.

in the UL-OCP. For each value of $\Delta \bar{t}$, we record the cost of operation over the entire simulation scenario, as well as the time to solve the UL-OCP. Fig. 4 reports the simulation results.
For $\Delta \bar{t}=5 \mathrm{~s}$ and $\Delta \bar{t}=10 \mathrm{~s}$, the hierarchical algorithm obtains the same solution as Gurobi. This means that the cost increase is $0 \%$. As the sampling time increases, the computation time decreases and the cost of operation increases. In the extreme case, $\Delta \bar{t}=900 \mathrm{~s}$, the relative cost increase compared to direct solution of the OCP is $33 \%$. For $\Delta \bar{t}=60 \mathrm{~s}$, the cost increase is less than $1 \%$, while the computation time is reduced by two orders of magnitude. We conclude that the binary decision variables, (3), can be determined efficiently by solving the UL-OCP on a coarse temporal time-scale, without a significant increase in the cost of operation.

The average computation time to solve the LL-OCP with $\tilde{N}=N=2160$ is $3 \mathrm{~s}$. This is critical, as the LL-OCP is solved as part of the (high frequency) lower optimization level in Algorithm 1. We therefore fix the sampling time to $\Delta \bar{t}=30$ $\mathrm{s}$ in the UL-OCP, and vary the horizon length $\tilde{N}$ in the LLOCP. Fig. 5 shows the average computation time to solve the LL-OCP and the cost of operation, as a function of $\tilde{N}$. For small $\tilde{N}$, the cost is significantly larger than for $\tilde{N}=2160$. When $\tilde{N}=64$, the cost increase is less than $5 \%$, and for $\tilde{N}=128$ the cost increase is less than $1 \%$. Compared to the case where $\tilde{N}=N=2160$, the computation time to solve the LL-OCP is reduced by one order of magnitude for $\tilde{N}=64$. 


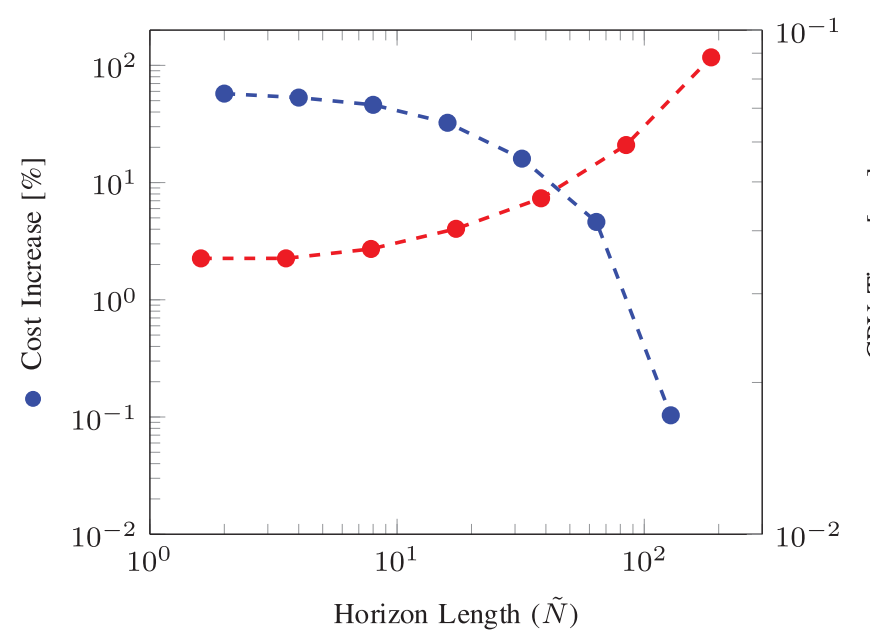

Fig. 5. Relative cost increase and average computation time to solve the LL-OCP with $\Delta t=5 \mathrm{~s}$, as a function of the prediction horizon $\tilde{N}$.

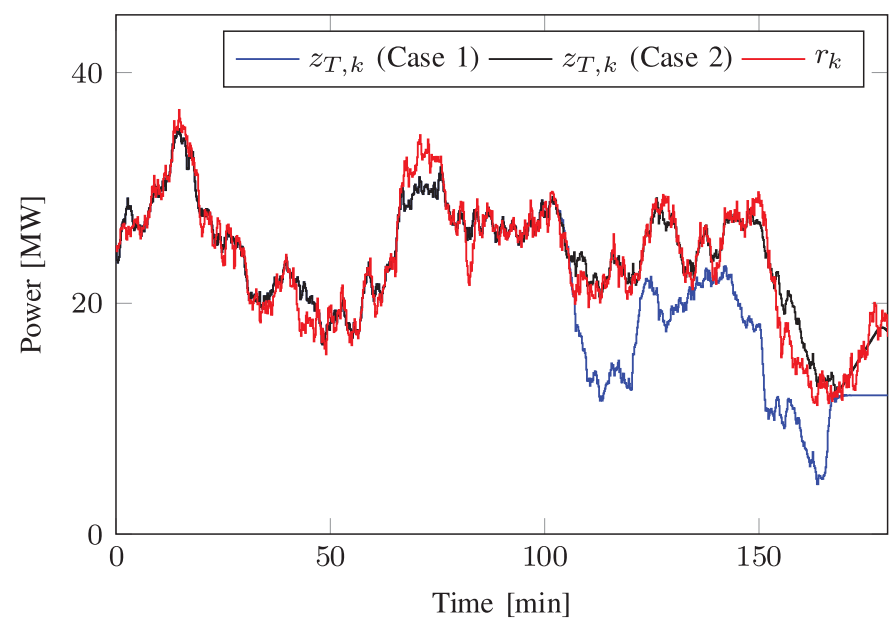

Fig. 6. Closed-loop simulation: Total power production. In Case 1, the binary scheduling variables are only updated at the beginning of the simulation. In Case 2, the binary scheduling variables are also updated at time step $k=900$ using the updated forecast $\hat{d}_{k \mid 900}$.

For $\Delta \bar{t}=60 \mathrm{~s}$ and $\tilde{N}=128$, the UL-OCP is solved in $2 \mathrm{~s}$ and the LL-OCP is solved in $0.1 \mathrm{~s}$. In comparison, the time to solve the OCP (7) directly is approximately $15 \mathrm{~min}$.

\section{B. Unknown Disturbance}

Consider the more realistic case where only forecasts of the disturbance, $\boldsymbol{d}_{k}$, are available. Two closed-loop simulations are performed. In the first simulation (Case 1), the UL-OCP is solved one single time using the initial forecast, $\hat{d}_{k \mid 0}$. In the second simulation (Case 2), the UL-OCP is resolved at time step $k=900$ using the updated forecast, $\hat{d}_{k \mid 900}$. The parameter specifications for Algorithm 1 are $\Delta \bar{t}=60 \mathrm{~s}$ and $\tilde{N}=128$.

We assume that a perfect disturbance forecast is available in the LL-OCP. This means that $\boldsymbol{d}_{k}$ is known 10 min ahead of time for the LL-OCP. Fig. 6 shows the total power production for Case 1 and for Case 2. In Case 1, significant deficits in the total power production occur for $t \geq 75 \mathrm{~min}$. This is because two of the three generators are turned off for $t \geq 75 \mathrm{~min}$, as a result of the binary scheduling decisions made at time $t=0$ min. As the binary variables are fixed in the LL-OCP, the generators cannot be turned on. For Case 2, the power deficits are avoided. Based on the updated forecast, $\hat{d}_{k \mid 900}$, the ULOCP modifies the initial plan to have more generators turned on for $t \geq 75 \mathrm{~min}$. This reduces costs by $75 \%$ compared to Case 1. In practice, the UL-OCP may be solved e.g. every 5 min using the most recent forecasts. It is important to note that this is only possible using the hierarchical algorithm.

\section{CONCLUSIONS}

In this paper, we have developed a hierarchical algorithm for MPC of a subclass of hybrid systems. The algorithm decomposes the OCP into an upper level MILP and a lower level LP. Binary scheduling variables are determined by solving the upper level MILP, and continuous control variables are determined by solving the lower level LP. The binary variables occur as fixed parameters in the lower level LP. The proposed approach reduces the most time-critical numerical operations in solving the OCP to solution of the lower level LP and allows frequent solution of the upper level MILP. The performance of the hierarchical algorithm was tested using a power portfolio case study. For this case study, the computation time to solve the open-loop OCP using the hierarchical algorithm is in the order of seconds. In comparison, the time to solve the OCP directly using a state-of-the-art MILP solver is more than 15 minutes. The performance improvement in computation time is achieved at the expense of less than a $1 \%$ increase in the MILP objective function.

\section{ACKNOWLEDGEMENT}

This work was funded in part by 1) the Danish Ministry of Higher Education and Science in the industrial $\mathrm{PhD}$ project "Stochastic MPC with Applications in Smart Energy Systems" (11-117435); 2) the Southern Denmark Growth Forum and the European Regional Development Fund in the project Smart \& Cool (ERDFD-10-0083); and 3) the Danish Council for Strategic Research in the project "CITIES - Centre for ITIntelligent Energy Systems in Cities" (1305-00027B).

\section{REFERENCES}

[1] J. B. Rawlings, "Tutorial Overview of Model Predictive Control," IEEE Control Systems, vol. 20, no. 3, pp. 38-52, 2000.

[2] D. Q. Mayne, J. B. Rawlings, C. V. Rao, and P. O. M. Scokaert, "Constrained Model Predictive Control: Stability and Optimality," $\mathrm{Au}$ tomatica, vol. 36, no. 6, pp. 789-814, 2000.

[3] J. B. Rawlings and D. Q. Mayne, Model Predictive Control: Theory and Design. Nob Hill Publishing, 2009.

[4] F. Borrelli, A. Bemporad, and M. Morari, Predictive Control for Linear and Hybrid Systems. Cambridge University Press, 2015.

[5] R. Amrit, J. B. Rawlings, and L. T. Biegler, "Optimizing process Economics Online Using Model Predictive Control," Computers \& Chemical Engineering, vol. 58, pp. 334-343, 2013.

[6] F. Borrelli, A. Bemporad, M. Fodor, and D. Hrovat, "An MPC/Hybrid System Approach to Traction Control," IEEE Transactions on Control Systems Technology, vol. 14, no. 3, pp. 541-552, 2006.

[7] L. F. S. Larsen, T. Geyer, and M. Morari, "Hybrid Model Predictive Control in Supermarket Refrigeration Systems," in 16th IFAC World Congress, 2005.

[8] D. Sarabia, F. Capraro, L. F. S. Larsen, and C. De Prada, "Hybrid NMPC of Supermarket Display Cases," Control Engineering Practice, vol. 17, no. 4, pp. 428-441, 2009. 
[9] L. F. S. Larsen, R. Izadi-Zamanabadi, and R. Wisniewski, "Supermarket Refrigeration System - Benchmark for Hybrid System Control," in European Control Conference (ECC), 2007, pp. 113-120.

[10] G. Ferrari-Trecate, E. Gallestey, P. Letizia, M. Spedicato, M. Morari, and M. Antoine, "Modeling and Control of Co-Generation Power Plants: A Hybrid System Approach," IEEE Transactions on Control Systems Technology, vol. 12, no. 5, pp. 694-705, 2004.

[11] J. L. Villa, M. Duque, A. Gauthier, and N. Rakoto-Ravalontsalama "Supervision and Optimal Control of a Class of Industrial Processes," in IEEE Conference on Emerging Technologies and Factory Automation, vol. 2, 2003, pp. 177-180.

[12] E. Mestan, M. Türkay, and Y. Arkun, "Optimization of Operations in Supply Chain Systems using Hybrid Systems Approach and Model Predictive Control," Industrial \& Engineering Chemistry Research, vol. 45, no. 19, pp. 6493-6503, 2006.

[13] A. Bemporad, F. Borrelli, and M. Morari, "Optimal Controllers for Hybrid Systems: Stability and Piecewise Linear Explicit Form," in 39th IEEE Conference on Decision and Control (CDC), vol. 2, 2000, pp. $1810-1815$.

[14] A. Bemporad, "Explicit Model Predictive Control," in Encyclopedia of Systems and Control, J. Baillieul and T. Samad, Eds. Springer London, 2014, pp. 1-9.

[15] D. Axehill, T. Besselmann, D. M. Raimondo, and M. Morari, "A Parametric Branch and Bound Approach to Suboptimal Explicit Hybrid MPC," Automatica, vol. 50, no. 1, pp. 240-246, 2014.

[16] D. Axehill and A. Hansson, "A Dual Gradient Projection Quadratic Programming Algorithm Tailored for Model Predictive Control," in 47th IEEE Conference on Decision and Control (CDC), 2008, pp. 3057-3064.

[17] C. Kirches, "Fast Numerical Methods for Mixed-Integer Nonlinear Model-Predictive Control," Ph.D. Thesis, Ruprecht-KarlsUniversität Heidelberg, 2010. [Online]. Available: http://archiv.ub.uniheidelberg.de/volltextserver/11636

[18] A. Bemporad, W. P. M. H. Heemels, and B. De Schutter, "On Hybrid Systems and Closed-Loop MPC Systems," IEEE Transactions on Automatic Control, vol. 47, no. 5, pp. 863-869, 2002.

[19] M. Lazar, W. P. M. H. Heemels, S. Weiland, and A. Bemporad, "Stabilizing Model Predictive Control of Hybrid Systems," IEEE Transactions on Automatic Control, vol. 51, no. 11, pp. 1813-1818, 2006.

[20] M. Lazar and W. P. M. H. Heemels, "Predictive Control of Hybrid Systems: Input-to-State Stability Results for Sub-Optimal Solutions," Automatica, vol. 45, no. 1, pp. 180-185, 2009.

[21] A. Bemporad, D. Mignone, and M. Morari, "Moving Horizon Estimation for Hybrid Systems and Fault Detection," in American Control Conference (ACC), vol. 4, 1999, pp. 2471-2475.

[22] M. Morari and M. Barić, "Recent Developments in the Control of Constrained Hybrid Systems," Computers \& Chemical Engineering, vol. 30, no. 10-12, pp. 1619-1631, 2006.

[23] M. Morari, M. Baotic, and F. Borrelli, "Hybrid Systems Modeling and Control," European Journal of Control, vol. 9, no. 2-3, pp. 177-189, 2003.

[24] J. Lunze and F. Lamnabhi-Lagarrigue, Handbook of Hybrid Systems Control: Theory, Tools, Applications. Cambridge University Press, 2009.

[25] F. W. Vaandrager and J. H. Van Schuppen, "Verification of Hybrid Systems via Mathematical Programming," in Hybrid Systems: Computation and Control, ser. Lecture Notes in Computer Science. Springer Berlin Heidelberg, 1999, vol. 1569, pp. 31-45.

[26] O. Slupphaug and B. A. Foss, "Model Predictive Control for a Class of Hybrid Systems," in European Control Conference (ECC), 1997.

[27] P. Rivotti and E. N. Pistikopoulos, "A Dynamic Programming Based Approach for Explicit Model Predictive Control of Hybrid Systems," Computers \& Chemical Engineering, vol. 72, pp. 126-144, 2015.

[28] D. Axehill, L. Vandenberghe, and A. Hansson, "Convex Relaxations for Mixed Integer Predictive Control," Automatica, vol. 46, no. 9, pp. $1540-1545,2010$

[29] M. Schmitt, R. Vujanic, J. Warrington, and M. Morari, "An Approach for Model Predictive Control of Mixed Integer-Input Linear Systems Based on Convex Relaxations," in 52nd IEEE Conference on Decision and Control (CDC), 2013, pp. 6428-6433.

[30] A. G. Beccuti, T. Geyer, and M. Morari, "Temporal Lagrangian Decomposition of Model Predictive Control for Hybrid Systems," in 43rd IEEE Conference on Decision and Control (CDC), vol. 3, 2004, pp. 2509-2514.

[31] L. Zukui and M. G. Ierapetritou, "Production Planning and Scheduling Integration through Augmented Lagrangian Optimization," Computers \& Chemical Engineering, vol. 6, no. 34, pp. 996-1006, 2010.
[32] M. Baldea and I. Harjunkoski, "Integrated Production Scheduling and Process Control: A Systematic Review," Computers \& Chemical Engineering, vol. 71, pp. 377-390, 2014

[33] J. Du, J. Park, I. Harjunkoski, and M. Baldea, "A Time Scale-Bridging Approach for Integrating Production Scheduling and Process Control," Computers \& Chemical Engineering, vol. 79, pp. 59-69, 2015.

[34] M. Soroush and D. J. Chmielewski, "Process Systems Opportunities in Power Generation, Storage and Distribution," Computers \& Chemical Engineering, vol. 51, pp. 86-95, 2013.

[35] S. Engell and I. Harjunkoski, "Optimal Operation: Scheduling, Advanced Control and their Integration," Computers \& Chemical Engineering, vol. 47, pp. 121-133, 2012.

[36] J. Zhuge and M. G. Ierapetritou, "An Integrated Framework for Scheduling and Control Using Fast Model Predictive Control," AIChE Journal, vol. 61, no. 10, pp. 3304-3319, 2015.

[37] R. L. Tousain, "Dynamic Optimization in Business-Wide Process Control," Ph.D. Thesis, Delft University of Technology, 2002. [Online]. Available: http://repository.tudelft.nl

[38] L. Messner, H. Gattringer, and H. Bremer, "Efficient Online Computation of Smooth Trajectories Along Geometric Paths for Robotic Manipulators," in Multibody System Dynamics, Robotics and Control, H. Gattringer and J. Gerstmayr, Eds. Springer Vienna, 2013, pp. 1730.

[39] K. B. Reed, V. Kallem, R. Alterovitz, K. Goldberg, A. M. Okamura, and N. J. Cowan, "Integrated Planning and Image-Guided Control for Planar Needle Steering," in IEEE/RAS-EMBS International Conference on Biomedical Robotics and Biomechatronics, 2008, pp. 819-824.

[40] T. E. Marlin and A. N. Hrymak, "Real-Time Operations Optimization of Continuous Processes," in Chemical Process Control-V, 1996, pp. $156-164$.

[41] M. L. Darby, M. Nikolaou, J. Jones, and D. Nicholson, "RTO: An Overview and Assessment of Current Practice," Journal of Process Control, vol. 21, no. 6, pp. 874-884, 2011.

[42] G. Francois, S. Costello, and B. Dominique, "Application of RealTime Optimization Methods to Energy Systems in the Presence of Uncertainties and Disturbances," TMC Academic Journal, vol. 9, no. 2, pp. 19-40, 2015.

[43] S. Engell, "Feedback Control for Optimal Process Operation," Journal of Process Control, vol. 17, no. 3, pp. 203-219, 2007.

[44] L. E. Sokoler, "Methods and Algorithms for Economic MPC in Power Production Planning," Ph.D. Thesis, Department of Applied Mathematics and Computer Science, Technical University of Denmark, 2016. [Online]. Available: http://orbit.dtu.dk

[45] T. G. Hovgaard, K. Edlund, and J. B. Jørgensen, "The Potential of Economic MPC for Power Management," in 49th IEEE Conference on Decision and Control (CDC), 2010, pp. 7533-7538.

[46] K. Edlund, J. D. Bendtsen, and J. B. Jørgensen, "Hierarchical ModelBased Predictive Control of a Power Plant Portfolio," Control Engineering Practice, vol. 19, no. 10, pp. 1126-1136, 2011.

[47] M. Carrión and J. M. Arroyo, "A Computationally Efficient MixedInteger Linear Formulation for the Thermal Unit Commitment Problem," IEEE Transactions on Power Systems, vol. 21, no. 3, pp. 1371-1378, 2006.

[48] A. J. Wood and B. F. Wollenberg, Power Generation, Operation, and Control, 3rd ed. John Wiley \& Sons, 2013

[49] V. Spudić, M. Jelavić, M. Baotić, and N. Perić, "Hierarchical Wind Farm Control for Power/Load Optimization," in Torque, 2010, pp. 681-692.

[50] J. J. Siirola and T. F. Edgar, "Process Energy Systems: Control, Economic, and Sustainability Objectives," Computers \& Chemical Engineering, vol. 47, pp. 134-144, 2012.

[51] L. Grüne, "Economic Receding Horizon Control without Terminal Constraints," Automatica, vol. 49, no. 3, pp. 725-734, 2013.

[52] K. Worthmann, "Estimates of the Prediction Horizon Length in MPC: A Numerical Case Study," in 4th IFAC Nonlinear Model Predictive Control Conference, 2012, pp. 232-237.

[53] A. Jadbabaie and J. Hauser, "On the Stability of Receding Horizon Control with a General Terminal Cost," IEEE Transactions on Automatic Control, vol. 50, no. 5, pp. 674-678, 2005.

[54] L. E. Sokoler, L. Standardi, K. Edlund, N. K. Poulsen, H. Madsen, and J. B. Jørgensen, "A Dantzig-Wolfe Decomposition Algorithm for Linear Economic Model Predictive Control of Dynamically Decoupled Subsystems," Journal of Process Control, vol. 24, no. 8, pp. 1225-1236, 2014.

[55] K. Edlund, T. Mølbak, and J. D. Bendtsen, "Simple Models for ModelBased Portfolio Load Balancing Controller Synthesis," in 6th IFAC Symposium on Power Plants and Power Systems Control, 2009, pp. 173178 . 


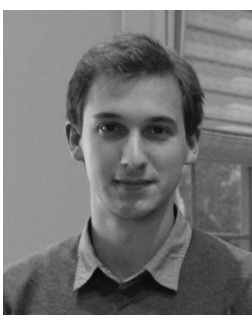

Leo Emil Sokoler received the (honors) M.Sc. degree in industrial mathematics from the Technical University of Denmark (DTU), Kgs. Lyngby, Denmark, in 2012. He received the Ph.D. degree in applied mathematics in 2016 at the Department of Applied Mathematics and Computer Science of DTU. The Ph.D. project was conducted in a collaboration between DTU and the Danish utility company DONG Energy. His research interests include convex optimization, power system modeling, and model predictive control.

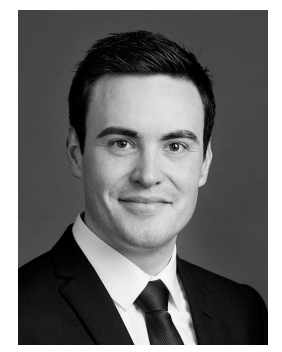

Peter Juhler Dinesen received the (honors) M.Sc degree in industrial mathematics from the Technical University of Denmark (DTU), Kgs. Lyngby, Denmark, in 2015. He is currently a management consultant at PA Consulting Group, Copenhagen, Denmark, working with process optimization, large data analysis, and project management. He combines his experiences within mathematical optimization modeling and consultancy to solve complex reallife challenges in various industries. His research interests include applied mathematical modeling, scientific computing, numerical optimization, and model predictive control.

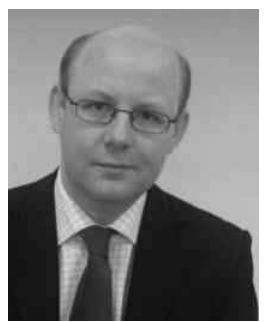

John Bagterp Jørgensen received the M.Sc. in engineering from the Technical University of Denmark (DTU), Kgs. Lyngby, Denmark, in 1997. He received the Ph.D. degree in chemical engineering in 2005 at the Department of Chemical Engineering of DTU. John Bagterp Jørgensen is an associate professor at the Department of Applied Mathematics and Computer Science of DTU. He is also a faculty member at DTU's Center for Energy Resources Engineering (CERE). His research concentrates on optimization and model predictive control including computational aspects and applications. The applications include industrial processes, intelligent control of smart energy systems, production optimization and closed-loop reservoir management of oil fields, and an artificial pancreas for people with type 1 diabetes. His research is to a large extent conducted in collaboration with industrial companies. He is also a co-founder of and a partner in 2-control ApS and has significant experience in online optimization and control of industrial processes. 